\begin{tabular}{|c|c|}
\hline Title: & $\begin{array}{l}\text { Measuring and Characterization of a Pedal Electric Cycle (Pedelec) on a Full System } \\
\text { Test-Bench with Full Range Emulation of a Cyclist }\end{array}$ \\
\hline Authors: & Michael Schmitt, Simon Decker, Martin Doppelbauer \\
\hline Institute: & $\begin{array}{l}\text { Karlsruhe Institute of Technology (KIT) } \\
\text { Institute of Electrical Engineering (ETI) }\end{array}$ \\
\hline Type: & Conference Proceedings \\
\hline Published at: & $\begin{array}{l}\text { Proceedings } 2019 \text { European Conference on Power Electronics and Applications } \\
\text { (EPE'19 ECCE Europe), Genova, Italy, } 2019 \\
\text { Publisher: IEEE } \\
\text { Year: } 2019 \\
\text { ISBN: } 978-9-0758-1530-6\end{array}$ \\
\hline Hyperlinks: & DOI: $10.23919 / E P E .2019 .8915567$ \\
\hline
\end{tabular}

(c) 2019 IEEE. Personal use of this material is permitted. Permission from IEEE must be obtained for all other uses, in any current or future media, including reprinting/republishing this material for advertising or promotional purposes, creating new collective works, for resale or redistribution to servers or lists, or reuse of any copyrighted component of this work in other works. 


\title{
Measuring and Characterization of a Pedal Electric Cycle (Pedelec) on a Full System Test-Bench with Full Range Emulation of a Cyclist
}

\author{
Michael Schmitt, Simon Decker, Martin Doppelbauer \\ Karlsruhe Institute of Technology \\ Institute of Electrical Engineering (ETI) \\ Kaiserstr.12 \\ Karlsruhe, Germany \\ Tel.: +49 721/608-41907 \\ E-Mail: M.Schmitt@kit.edu \\ URL: http://www.eti.kit.edu
}

\section{Keyword}

$<<$ Hybrid Electric Vehicle $(\mathrm{HEV})>>,<<$ Test bench $>>,<<$ Measurement $>>$

\begin{abstract}
This paper describes the development of a pedelec test-bench and the measurement of a pedelec to characterize the propulsion system. The main idea of the test-bench is to emulate a real driver more precisely then other state of the art test-benches. This means precise, bilateral pedal torque inducement and direct emulation of the road by a roller. Precise measurement equipment and dynamic control enables versatile measurements with the proposed test-bench. Different measurements are introduced amongst others: assistance level, dynamic control behavior, distance and efficiency with full range emulation of a cyclist.
\end{abstract}

\section{Introduction}

Focused on new urban mobility concepts, comfortable and simple mobility, healthy and rehabilitation in sport or entering new dimensions in cycling, more and more different electric supported bikes (Ebikes) exist. A subcategory of these are pedelecs (pedal electric cycles). These bikes offer electric supported cycling in a low power range, normally without additional license or registration. Measurement of the assistance level and the admissible maximum output power of pedelecs is therefore essential. Furthermore, characterizations like long-range testing, assistance level mode, efficiency, regeneration mode, cutoff-time and dynamic control behavior for natural feeling of the pedelec are of interest to consumers. Similar to hybrid electric vehicles, full system test-benches with full range emulation are necessary for independent and reproducible measurement results. Commercial testbenches are designed for end of line tests or functional tests. Even special developed test-benches do not cover full range emulation of the cyclist. Often these systems are supplying only single side pedal torque inducement based on a constant torque, e.g. [1] and [2]. Further, they set a speed at the pedals and a torque at the roll. This leads to a constant and one-sided torque inducement at the pedals. For precise emulation the pedal torque of a cyclist, a sinusoidal torque, must be induced at both the pedals. Measurements with pulling and pushing pedal torque or even with faults like unsymmetrical torque inducement are interesting, too, but cannot be covered with state-of-the-art test-benches. In this paper, motivated by precision and full range emulation of a cyclist, a new test-bench is developed. In addition, test-bench measurements like the German ZIV R200 endurance/distance test [3], assistance level tests, etc. are applied.

Therefore, a cyclist is analyzed at first. Based on that, the power flow of a cyclist, the pedelec and the terrain, similar to hybrid electric vehicles is derived. Further, the requirements for a full range emulation are defined. Afterwards, the mechanic, electric, signal processing as well as the used control scheme is explained. Following, the device under test and the measurements are described to characterize the propulsion system of the pedelec. The summery end up this paper. 


\section{Modeling: Cyclist, Pedelec and Terrain}

Before the design of the test-bench started, a cyclist, the terrain and the pedelec are analyzed, and as well as the basic conditions defined. Based on the power flow diagram in Fig. 1, the requirements of maximum speed, maximum torque and the dynamics are deviated.

\section{Pedelec - Power flow}

The power flow of a cyclist on a pedelec is shown in Fig. 1. Thereby, three fundamental power parts must be considered: The power induced at the pedals of the cyclist in green, the power from the pedelec drivetrain in dark blue and the pedelec output power in light blue. The input power of the cyclist itself consists of two parts again, the input power of the left and the right leg. Together these builds the induced power. The power of the pedelec drivetrain depends on the cyclist's torque and cadence with pedelecmotor control algorithms considering e.g. the assistance level mode. These are characteristics of the different pedelec manufacturers. The output power is defined by the effective speed and torque at the rear wheel and is the power from the cyclist and the pedelec drivetrain on the road. Precise emulation of the cyclist and the terrain is thereby required for the ETI-Pedelec test-bench and analyzed in detail in the following.

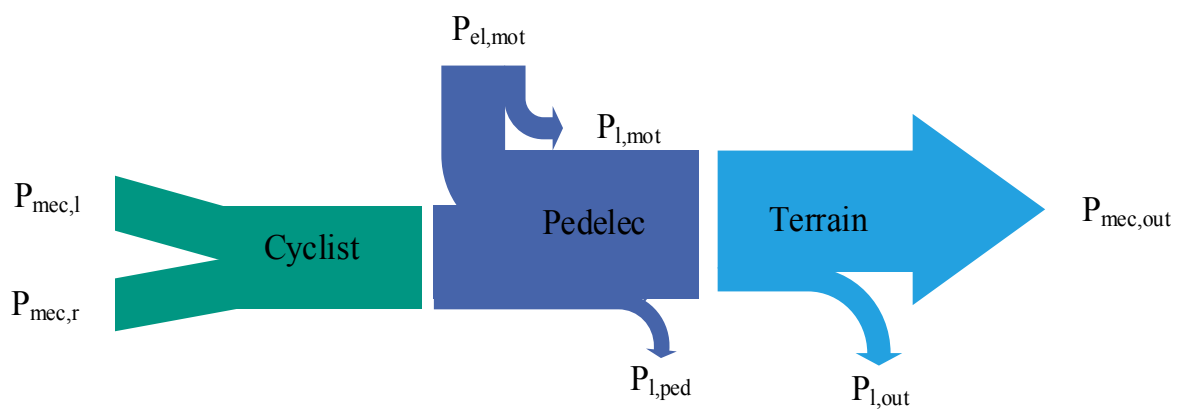

Fig. 1: Pedelec power flow diagram

\section{Cyclist}

Analysis of a typical cyclist shows, that the power at the pedals consists of two parts, one from the left and one from the right leg, Fig. 1. A cyclist induces a position-dependent torque to the pedal. The rotational speed of the crank results from the desired velocity and the selected gear of the cycle. For the test-bench, this implies that the cyclist should be emulated by two PSM's (permanent magnet synchronous machine) and these should be torque controlled.

The pedal torque of a cyclist is determined in detail in [4] and [5]. The torque can be simplified to the norm of a sinusoidal curve, as shown in Fig. 2. The sinusoidal shape is due to the mass of the cyclist, gravity, pedal rotation, pedal axis itself and the length of the crank. The crank behaves like a lever arm and is about $175 \mathrm{~mm}$ long. The maximum and minimum torque depends on the angel of the crank. The average torque of semi-trained cyclist is assumed to $40 \mathrm{Nm}$. The maximum torque $T_{\max }$ of a cyclist is up to $170 \mathrm{Nm}$ as shown in equation (1).

$$
\begin{aligned}
& T_{\max }=F_{\max } \cdot l=171 \mathrm{Nm} \\
& l=0.175 \mathrm{~m} \\
& F_{\max }=m \cdot a=100 \mathrm{~kg} \cdot 9.81 \frac{\mathrm{m}}{\mathrm{s}^{2}}=981 \mathrm{~N}
\end{aligned}
$$




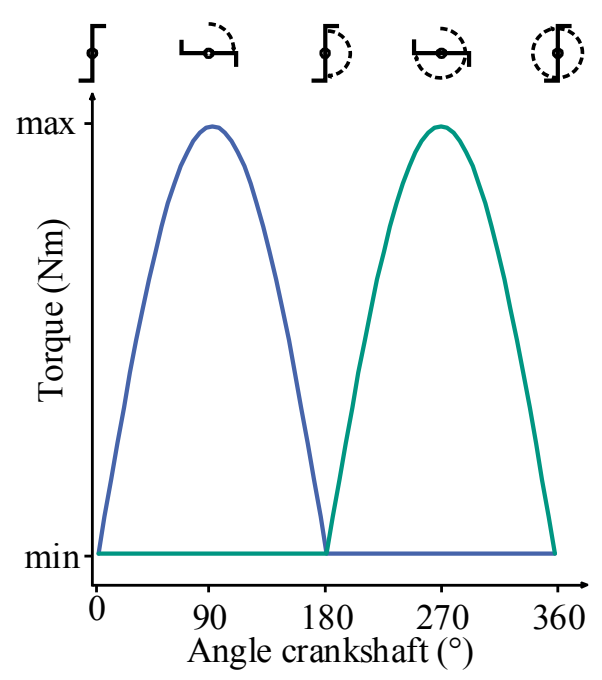

Fig. 2: Approximated input torque

\section{Terrain}

For terrain emulation, a straight road with different slopes is assumed. Therefore, a roll in combination with a motor under load is applied. In the further course of this paper, this motor is called "load PSM". Controlling the rotational speed of the roll with the load PSM in balance with the induced pedal torque determines the explicit operation points. By adjusting the wheel vertical force, equal test-conditions are possible.

\section{Test-bench}

The developed ETI-Pedelec test-bench should emulate a real driver and the road precisely This means a sinusoidal torque on both pedals, as described in [4] and [5], have to be induced. Further, the roll has to be speed controlled precisely. The control, the evaluation of the measurements results, the construction, the topology are challenging. Therefore, a more detailed view is shown in the following

\section{Topology}

In Fig. 3, the full electrical topology of the ETI-pedelec test-bench is shown. Two PSM's emulate the cyclist and the terrain is emulated by one PSM. All PSM machines are from SEW type CMP71S with additional gears. Thereby, each PSM is applied by his own power converter. In a normal driving cycle, the roll operates in brake mode respectively the load PSM operates as a generator. The PSMs at the pedals operate in motor mode. With a shared DC link of all converters, the grid connected active frontend (AFE) only covers the losses.

\section{Hardware / Software}

The modular power electronic converters are based on the IGBT modules from Infineon type EconoPack FS75R12KT4. The switching frequency of the converters are $8 \mathrm{kHz}$. Each of these converters is equipped with voltage, current and thermal sensors. The motors are equipped with resolvers for precise measurement of the angle and rotational speed. Two precise torque sensors are used to measure the torque at one pedal PSM (PSM at the pedal) and one at the load PSM. An external measurement board is used to measure battery voltage and current. To quantify the rear wheels vertical force, a force sensor is applied. Each power electronic converter is equipped with voltage, current and thermal sensors to supervise and control the PSM.

The control and monitoring of the ETI-Pedelec test-bench is realized by means of two self-developed signal processing systems with digital signal processors (DSPs), Analog-Digital (A/D) conversion cards, angular evaluation cards. These DSP systems thereby monitor and control the test-bench and operates with $8 \mathrm{kHz}$. The system is presented in detail in [6].

The first of the two DSP-systems is responsible for the AFE and output motor. The second is used for the two pedal PSMs. Both signal-processing systems are linked together. The control software for the 
ETI-Pedelec test-bench is implemented in Matlab/Simulink and adopted to the DSP via Simulink's automatic code generation. The signal processing systems are connected via USB interface to a computer. The computer with the LabVIEW monitoring tool is the human-machine interface (HMI). It is used to serve the test-bench, to monitor the measured and internal values as well as for automatic measurement routines.

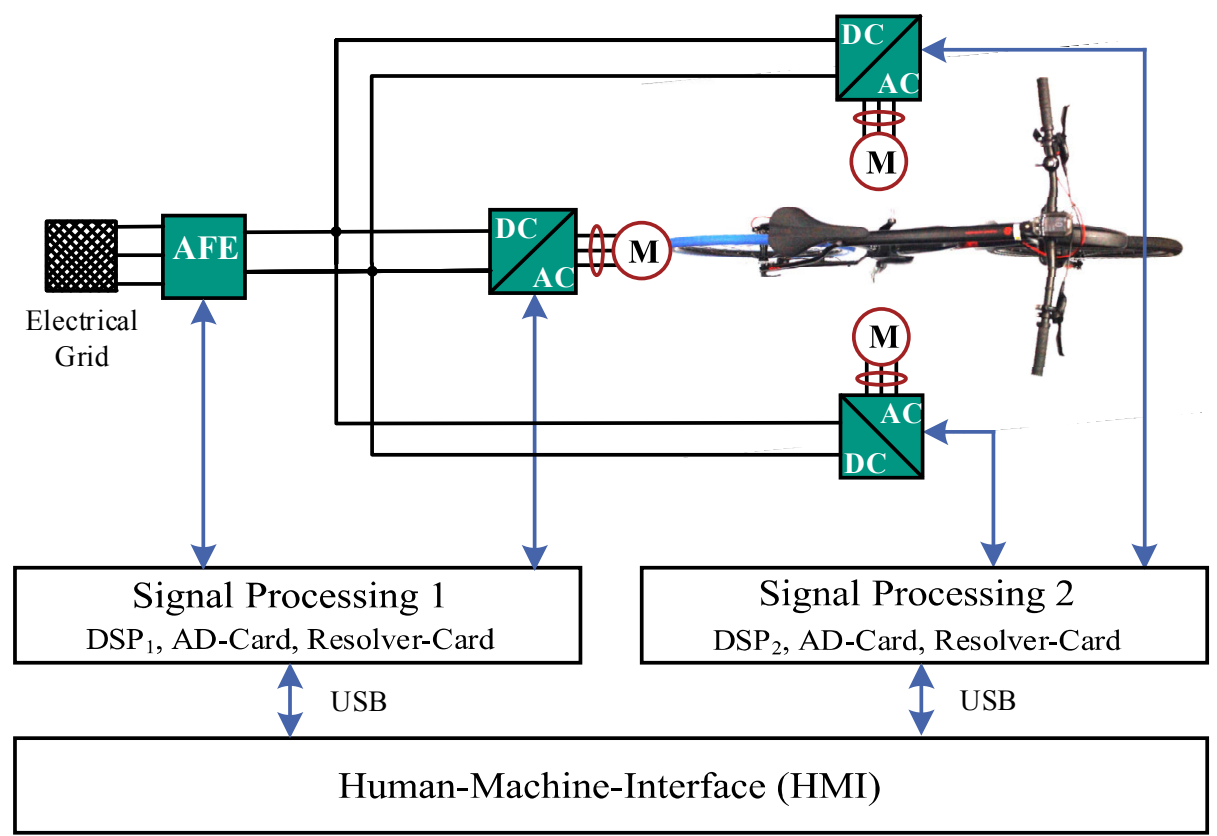

Fig. 3: Topology of the ETI-pedelec test-bench

\section{Control}

As already described, the pedal PSMs, which emulates the cyclist, must induce a position-dependent torque. Thus, the load PSM must be speed controlled.

At first, the control scheme for the two pedal PSMs is described. A control period is thereby $125 \mu \mathrm{s}$. The pedal PSM are inducing the torque according to Fig. 2. Because of drag in the PSMs, a constant offset $T_{\mathrm{An} \text {,const }}$ is necessary, to avoid a dead center point. The overall torque consists of the sinusoidal part and this constant part. Thereby one pedal PSM has to induce one sinusoidal half wave and a constant part. The blue curve must be induced at one pedal PSM as seen in Fig. 2. The sinusoidal half waves of the two pedal PSM are $180^{\circ}$ phase shifted. The control consists of an overlaying torque controller and an underlying current controller. The overlaying torque control is implemented in an open control loop; the required dynamics cannot be achieved with a closed feedback system. The cut-off frequency of the torque measurement is within the operation area. The control structure of one pedal PSM is shown in Fig. 4.

The desired constant torque and driver torque are set via the HMI. The maximum torque is thereby limited to protect the device under test (DUT). In look up table $1\left(\mathrm{LUT}_{1}\right)$ the form of the torque is stored depending on the angle of the crank. Therefore, the torque has its sinusoidal shape after the limitation, as described above. Additionally, any other shape of the torque is possible, like push and pull or constant torque. After $\mathrm{LUT}_{1}$, the constant part of the torque is added to obtain the total torque. Furthermore, $\mathrm{LUT}_{2}$ is required due to the open loop control. It determines the necessary currents for the desired torque from the actual speed. In order to obtain $\mathrm{LUT}_{2}$, both pedal PSM are characterized in steady-state measurement to ensure the correct current for the desired torque in test-bench operation.

The following current controller is designed with the optimum amount. The maximum current is limited to avoid damage to the PSMs or the test-bench. 


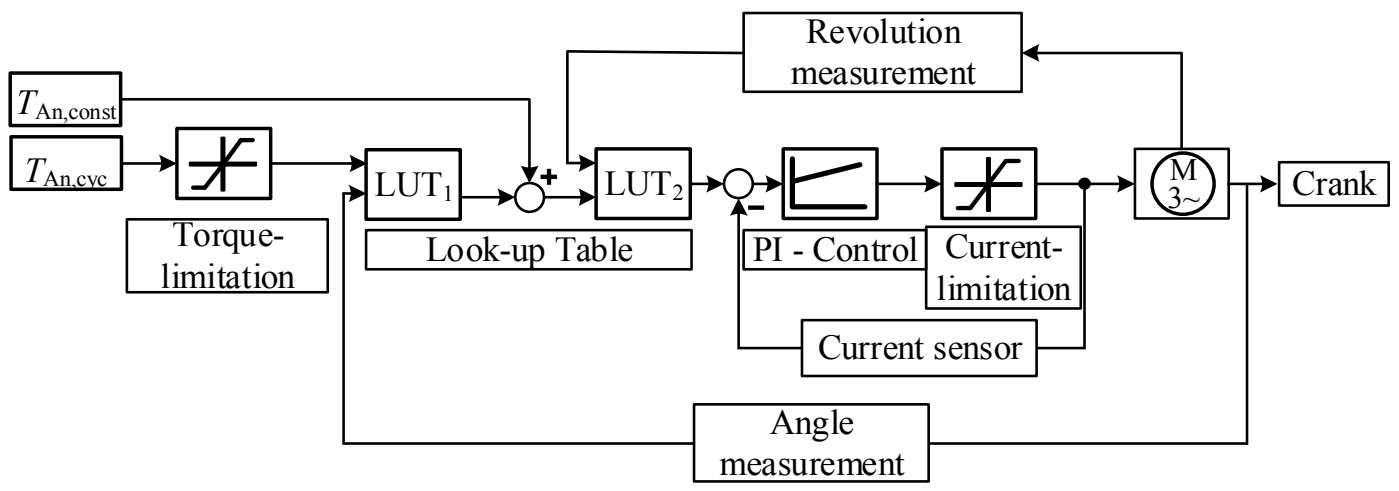

Fig. 4: Open loop circuit torque control of one pedal

The load PSM for terrain emulation by the roll is controlled in a cascaded control structure. The speed control is configured with the symmetrical optimum [7] and has an underlying current controller. Fig. 5 shows the scheme of the controller. The input of the controller is the desired speed $n_{\text {LAM }}$. A ramp generator prevents the speed from overshooting. A maximum speed limitation is implemented to avoid damage of the PSM or the test-bench. The first PI controller controls the speed and is configured with the symmetrical optimum. The underlying PI current controller is configured with the optimum amount similar to the pedal PSMs.

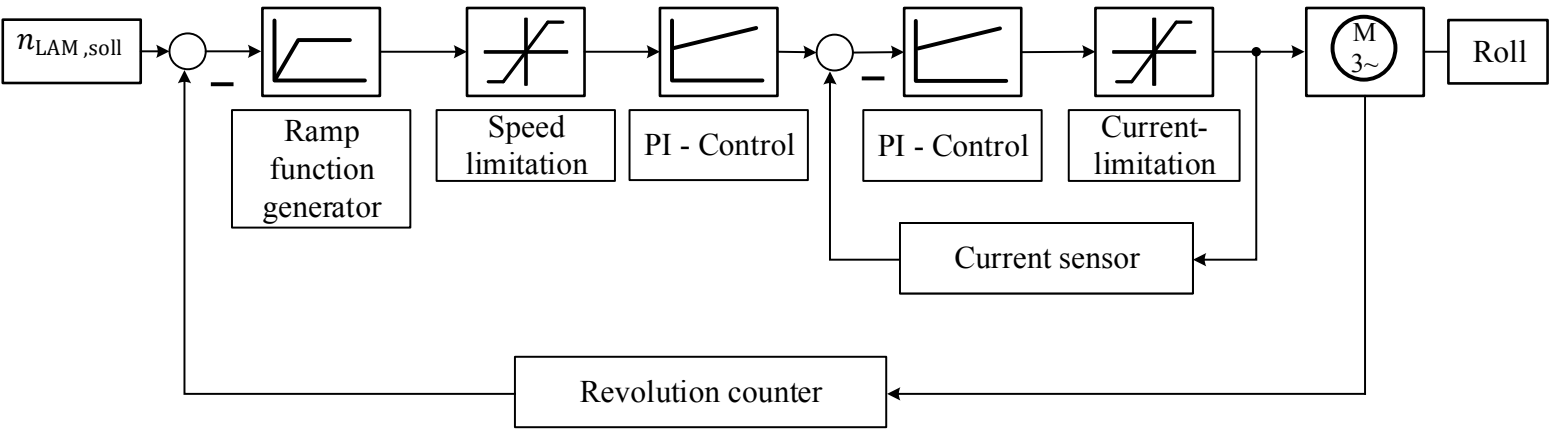

Fig. 5: Speed control of the load PSM

\section{Mechanic}

The mechanic is deviated from the power flow diagram and the resulting topology, shown in Fig. 3. In Fig. 6 the CAD-model of the mechanic of the ETI-Pedelec test-bench is shown. The test-bench can be separated in three parts. The first part is the rear wheel part with the load PSM. In the second part is the cyclist emulated and the third and last part is for the front wheel. The pedelec is thereby fixed with cargo straps into the test-bench. Additional weights and cargo straps are used to adjust the pressure from the rear wheel to the roll. The rear wheel stands on the roll and is fixed with another pair of brackets (part one). Thereby, the whole roll is mounted on a joint, with the additional force sensor, the wheel vertical force can be controlled. In the second part are the PSMs for the pedal torque mounted. The attachment of the PSMs by a tripod can be adjusted into three directions (up/down, left/right, and back/forth). Because of this, a wide range of pedelecs, with wheel sizes from 24" to 29", can be mounted and measured. The PSMs are connected to the crank and pedals are replaced with a universal bolt. The front wheel is fixed with a bracket on a platform. 


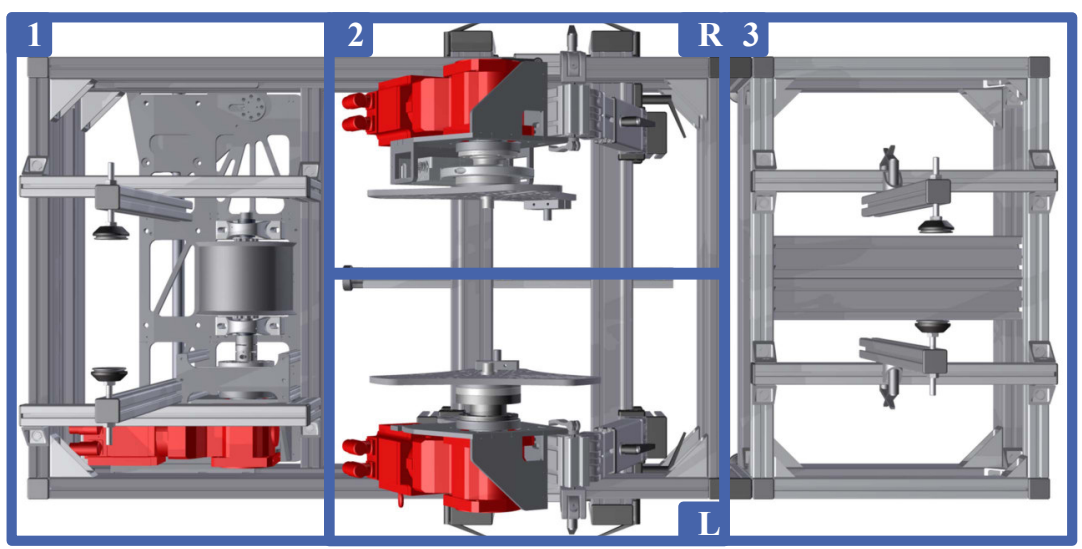

Fig. 6: Mechanic test-bench

\section{Device under test}

The device under test for the shown measurements is, a Ghost Kato 3 Hybrid pedelec. This pedelec is an up to date hardtail mountain bike with a middle motor. The pedelec is equipped with a Shimano E8000 motor, a $504 \mathrm{Wh}$ battery with $36 \mathrm{~V}$ and $14 \mathrm{Ah}$. The nominal power of the motor is $250 \mathrm{~W}$ and the maximum torque approx. $70 \mathrm{Nm}$. The mounted rear tire is a Tacx roller tire, which is used for constant long-term performance. Furthermore, slight test bench influences during operation on the roll can be neglected and comparable results can be guaranteed.

\section{Measurements}

The measurements in this paper focus on the overall system of a pedelec and should identify the characteristics of these and the associated motor with its controller under realistic conditions. For that, the test-bench emulates the real cyclist in special and typical cycling situations. To identify the drivetrain and the motor characteristics, several of these measurements are needed. The assistance level measurement identifies the reaction of the pedelec-motor in different cycling situations. On one side by increasing input torque at constant velocity and on the other side by increasing velocity at constant mean torque. The distance measurements characterize the maximum reachable range with one full loaded battery and the dynamics the start/stop and acceleration behavior of the device under test. Furthermore, the efficiency of the pedelec-motor, is calculated for each measurement using the measured values. These typical measurements including their necessary start-up procedures are shown in the following.

\section{Initialization}

It is important to choose the right gear for the measurements. For the assistance level measurement, the gear must be selected so that typical speeds of a cyclist are covered. This means the speed of the cyclist should be between 15 and $90 \mathrm{rpm}$ while the velocity of the pedelec is between 3 and $27 \mathrm{~km} / \mathrm{h}$.

For the distance measurement, the gear is selected as described in [3], so that the cyclist has a speed of about $60 \mathrm{rpm}$ at about $20 \mathrm{~km} / \mathrm{h}$.

Further, for comparable results a warm-up phase is required before starting the measurements. This is necessary because the warm-up of tires, bearings and PSMs, etc. lead to transient changes in the first minutes of the measurement. To minimize these effects, a warm-up phase of 30 minutes must be performed, after which it is assumed, that transient changes have no effect on the results. Almost constant losses of the loss detection routine of the test-bench before and after a distance measurement confirm this result.

\section{Distance}

The distance measurement quantifies the maximum reachable range when the battery is fully loaded. A new method to calculate this value is presented by the German ZIV (German bicycle industry association) and is defined in [3]. The aim of this method is to determine the maximum range under 
defined conditions and to normalize the assistance level to a uniform $200 \%$. Thus, the distance of different pedelecs from different manufacturers and types with different motors can be compared. For this measurement, the torque at the rear wheel and ultimately the distance achieved must be measured on the test-bench. In addition to these values required for measurement, the battery voltage and current of the pedelec-motor are measured over time to determine the efficiency.

The distance measurement is done with the Ghost Kato 3 hybrid, introduced before. According, to the test procedure, the tested pedelec reaches a range of about $17.3 \mathrm{~km}$ with a break force $F_{\mathrm{B}}$ of about $73.3 \mathrm{~N}$ on the test-bench. Using the formulas given in [3] results in a maximum range of about $53.9 \mathrm{~km}$ normalized to $200 \%$ assistance level. The energy efficiency is thereby about $9.4 \mathrm{Wh} / \mathrm{km}$. During the test, the pedelec consumes $376 \mathrm{Wh}$ or $75 \%$ of the nominal battery capacity. The remaining $25 \%$ are needed to prevent deep discharge of the battery. Fig. 7 shows the power curve of the cyclist, the battery and the output during the measurement. The power of the cyclist is about $71.3 \mathrm{~W}$. The measurement itself can be divided into three parts. During the first part, the first 8 minutes, the motor is able to operate at full power of about $565 \mathrm{~W}$. During the second part, the next 4 minutes, the motor reduces the power gradually due to possible derating of the motor. In the third part, the rest of the measurement, the motor power is constant at about $380 \mathrm{~W}$ and operates in the thermal equilibrium.

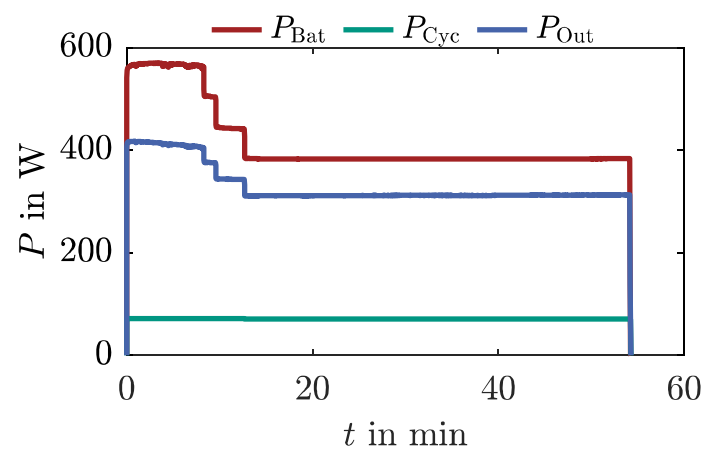

$$
\begin{gathered}
R_{200}=53.9 \mathrm{~km} \\
R_{\mathrm{P} 200}=40.8 \mathrm{~km} \\
R_{\mathrm{P}}=17.3 \mathrm{~km} \\
R=1.3 \\
N=2.4 \\
K=3.1 \\
P_{\text {Bat,Mean }}=417 \mathrm{~W} \\
\eta=68 \%
\end{gathered}
$$

Fig. 7: Course of power during distance measurement

\section{Assistance level}

Further measurements applied on this test-bench are the characterization of the assistance level which describes the support for the cyclist from the pedelec-motor. The assistance level (AL) is measured in different situations. The AL is calculated as described in equation (4). These situations are simulated, on the one hand, by varying the input torque at constant speed and on the other hand by varying the speed at constant torque. The measured variables are always the speed, torque at the rear wheel, cadence and torque at the crank. Thereby different assistance level modes are characterized by increasing input torque from $10 \mathrm{Nm}$ up to $40 \mathrm{Nm}$ at constant speed of about $20 \mathrm{~km} / \mathrm{h}$. The result is plotted in Fig. 8 and shows the AL of the "Boost" mode in blue, AL of the "Trail" mode in green and in red AL of the "Eco" mode. As a result, it can be seen that in "Boost" mode with increasing input torque, the assistance level is decreasing, due to the restricted power of the pedelec-motor. The assistance level of the "Trail" and "Eco" mode are quite similar. $T_{\mathrm{St}}$ is the stimulation torque of the cyclist. In Fig. 9, the same measurement is evaluated. In this figure, not the assistance level is shown, but the torque of the pedelec-motor at the rear wheel at increasing input torque. The figure shows, that the torque in the "Boost" mode is nearly constant at increasing input torque. In comparison, in "Trail" mode an increasing input torque yield to linearly increasing torque of the pedelec-motor. The "Eco" mode is restricted at about $28 \mathrm{Nm}$, for maximal battery endurance. 


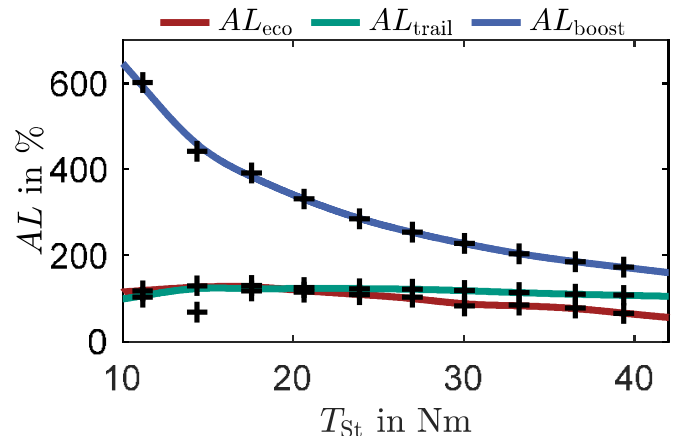

Fig. 8: Assistance level at increasing torque at about $20 \mathrm{~km} / \mathrm{h}$

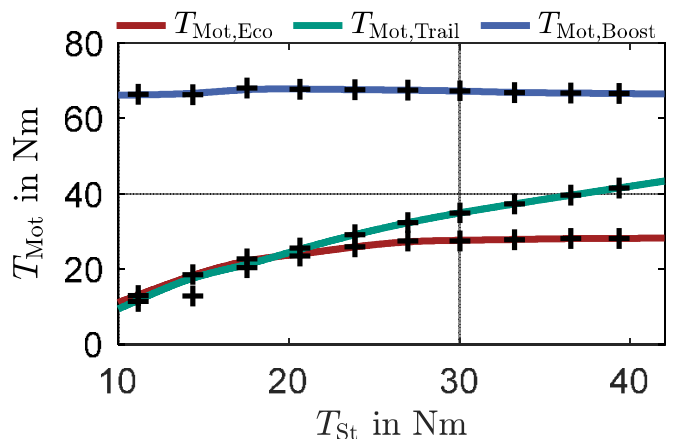

Fig. 9: Torque of the pedelec-motor at the roll at increasing input torque and constant speed

A second measurement is done at increasing speed and constant input torque. During the measurement, the input torque is set to $23 \mathrm{Nm}$. The speed is gradually increased from about $3 \mathrm{~km} / \mathrm{h}$ to $27 \mathrm{~km} / \mathrm{h}$. The result is shown in Fig. 10. The blue curve is the AL of the "Boost" mode, the green curve is the AL of the "Trail" mode and in red the AL of the "Eco" mode is shown. During speed increase, the AL is increasing slightly. After about $20 \mathrm{~km} / \mathrm{h}$ the AL starts to decrease, at about $25 \mathrm{~km} / \mathrm{h}$ the AL is close to $0 \%$. The AL of the "Boost" mode is very high with about $300 \%$. This means the power of the pedelec-motor is three times the power of the cyclist. The "Trail" mode is similar to the "Eco" mode at this little torque. The AL is about $100 \%$ to $120 \%$.

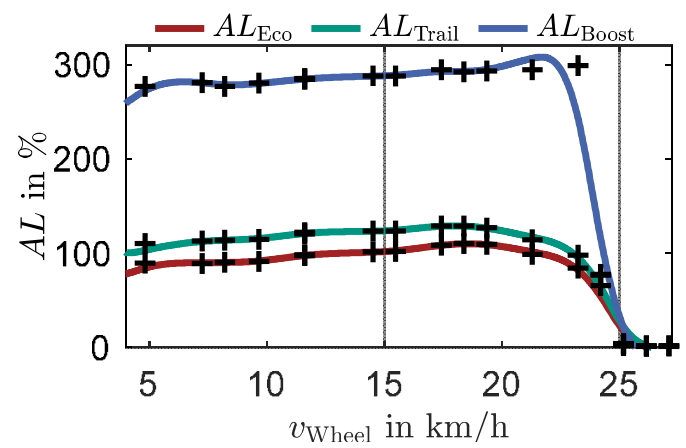

Fig. 10: Assistance level by increasing velocity at about $23 \mathrm{Nm}$

The definition of the assistance level described is given in equation (4). $F_{\mathrm{B}}$ is the total brake force at the rear wheel. $F_{\mathrm{D}}$ is the brake force at the rear wheel, caused by the cyclist.

$$
\mathrm{S}=\left(\frac{F_{\mathrm{B}}}{F_{\mathrm{D}}}-1\right) \cdot 100 \%
$$

\section{Dynamics}

Another interesting aspect is the feeling of riding a pedelec. This should be similar to a conventional bike and is very important to a cyclist. During a cycle ride, the conditions are changing permanently, for example the surface and the grip, or the slope of the road. A cyclist adopts to this intuitively and a pedelec must react similarly. This means, the cut-off time and the rise-time should be small and the torque changes should be smooth. The proposed ETI-Pedelec test-bench with its precise sensors is able to measure these dynamics. Emulation of acceleration of speed, increase or decrease of torque imposition enables the characterization of the dynamics. Fig. 11 shows exemplarily the acceleration of the pedelec from $5 \mathrm{~km} / \mathrm{h}$ to $10 \mathrm{~km} / \mathrm{h}$. In this measurement, the accelerations are performed at a constant torque so that only the speed changes. Since the gears are not changed, the cyclist has to cycle faster. This means that the cyclist's power is increasing and yields to increasing power of the pedelecmotor, for constant AL. This test-bench situation is used to simulate the rise-time of the pedelec-motor control during cycling. The easiest way to quantify this behavior is monitoring the battery current during the situation, because of almost constant battery voltage, the current increases as the power increases. 
This implies for the measurement, if the current of the battery increases by more than $10 \%$ during the measurement, the pedelec reacts to the acceleration of the test-bench. In the "Boost" mode, the pedelecmotor needs about $0.15 \mathrm{~s}$ to react to the acceleration from the test-bench.

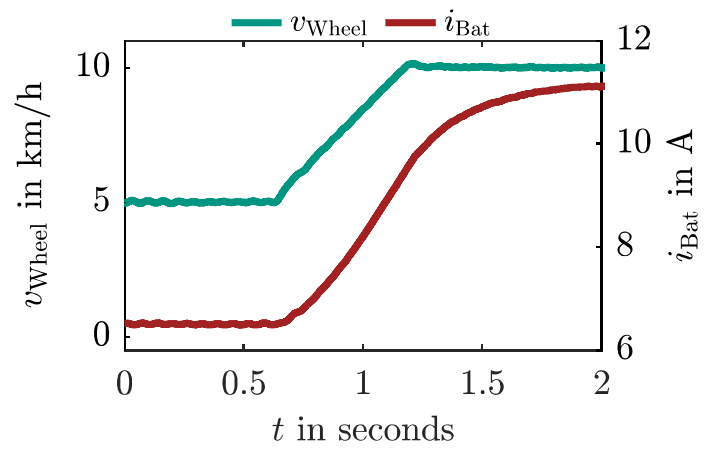

Fig. 11: Acceleration of the DUT from 5 to $10 \mathrm{~km} / \mathrm{h}$

\section{Further measurements}

As demonstrated before, it is possible to execute different measurements with this ETI-Pedelec testbench. The test-bench delivers precise results for standard as well as novel measurements. First of all, the topology can be altered by applying one only or two pedal PSM to the crank. Hence, the efficiency of the pedelec-motor can be calculated with these measurements by considering the different measured powers according the power flow diagram explained above.

In addition, the torque can be modified, e.g. push and pull or different amount of torque left and right. This means asymmetrical torque inducement, like it may happen in failure mode or when the cyclist is hurt can be emulated as well.

\section{Summary}

This paper presents a test bench for full range and real emulation of a cyclist. As it is state of the art in the development process of hybrid electric vehicles, independent and reproducible measurements can be performed. For this purpose, the presented ETI-Pedelec test-bench emulates a real driver more precisely than other state-of-the-art test benches. Several measurements can be performed to characterize the pedelec's propulsion system. Both the assistance level and the range of the pedelec can be measured. Furthermore, the dynamics, i.e. the reaction to a change in speed or torque, can be measured. Several failure modes such as asymmetrical or one-sided torque inducement can be simulated with the presented test bench. In addition, the curve of the torque can be changed, thus push and pull is possible as well. To archive this, a lot of sensors and a powerful signal processing hardware, modern control and measurement routines are used.

\section{References}

[1] velotech.de $\mathrm{GmbH}, \quad$ Test-Bench Velotech. [Online] Available: https://www.velotech.de/en/maximum-testing-accuracy-state-art-test-rigs. Accessed on: Jun. 05 2019.

[2] eChecker. Der Prüfstand für E-Bikes, Test-Bench eChecker. [Online] Available: https://www.ebike-checker.de/. Accessed on: Jun. 052019.

[3] Zweirad Industrie Verband (ZIV), Normierte Reichweite R200 für E-Bikes. [Online] Available: https://www.ziv-zweirad.de/presse/themen-dossiers/. Accessed on: May 082019.

[4] J.-C. Quintana-Duque, "Estimation of torque variation from pedal motion in cycling," 2015.

[5] P. Spagnol, M. Corno, and S. M. Savaresi, "Pedaling torque reconstruction for half pedaling sensor," in 2013 European Control Conference (ECC), Zurich, Jul. 2013 - Jul. 2013, pp. 275-280. 
[6] C. Axtmann, M. Boxriker, and M. Braun, "A custom, high-performance real time measurement and control system for arbitrary power electronic systems in academic research and education," in 2016 18th European Conference on Power Electronics and Applications (EPE'16 ECCE Europe): 5-9 Sept. 2016, Karlsruhe, 2016, pp. 1-7.

[7] D. Schröder, Elektrische Antriebe - Regelung von Antriebssystemen. Berlin, Heidelberg: Springer Berlin Heidelberg, 2015. 